\title{
Optimizing the Arrangement of Points on the Unit Sphere
}

\author{
By Joel Berman and Kit Hanes
}

\begin{abstract}
This paper is concerned with the problem of placing $N$ points on the unit sphere in $E^{3}$ so as to maximize the sum of their mutual distances. A necessary condition is proved which led to a computer algorithm. This in turn led to the apparent best arrangements for values of $N$ from 5 to 10 inclusive.
\end{abstract}

How does one place $N$ points on the surface of the unit sphere in $E^{3}$ so as to maximize the sum $S(N)$ of their mutual distances? This problem and its generalizations to $E^{m}$ have a rich and extensive literature. A sample is given in the references. For $m=2$ the solution is the regular $N$-gon with $\operatorname{sum} N \cot (\pi / 2 N)$ [6]. It is also known that the regular $N+1$ simplex inscribed in the unit sphere in $E^{N}$ is optimal. Thus, the regular tetrahedron gives $S(4)=9.79796$. For $N>4$ in $E^{3}$ the problem is still open.

In [3] a similar problem was considered: Place $N$ points on the surface of the sphere in $E^{3}$ so as to maximize the volume of their convex hull. The technique used was to find the best position for a point, relative to the other points, so as to maximize the volume function. This allowed for a complete solution of the problem up to $N=8$; and in particular, the solution for 8 points agreed with a candidate obtained by a computer search in [8]. An attempt to use this approach for the mutual distances problem leads to the following.

Lemma. Let $p_{1}, \ldots, p_{n}$ be points on the unit sphere $S$ in $E^{3}$. Let $f: S \rightarrow R$ be defined by $f(x)=\sum_{i=1}^{n}\left|x-p_{i}\right|$. If $f$ has a maximum at $p$, then $p=q /|q|$ where $q=\sum_{i=1}^{n}\left(p-p_{i}\right) /\left|p-p_{i}\right|$.

Proof. Since $\operatorname{grad} f(x)=\sum_{i=1}^{n}\left(x-p_{i}\right) /\left|x-p_{i}\right|$, then the normal to $S$ at $p$, that is, $p$ itself, must be a positive multiple of $\operatorname{grad} f(p)$, and the conclusion follows.

This lemma gives the position of the point $p$ as a function of $p$ itself. Nonetheless we have used this lemma as a basis for an iterative program whereby each point is repeatedly moved in an attempt to improve its position so as to satisfy the condition of the lemma. Naturally $p_{j}$ should be moved in the direction of

$$
q_{j}=\sum_{i \neq j}\left(p_{j}-p_{i}\right) /\left|p_{j}-p_{i}\right|
$$

We found that simply replacing $p_{j}$ by $q_{j} /\left|q_{j}\right|$ is adequate. Our results are summarized in Table 1.

Received December 14, 1976.

AMS (MOS) subject classifications (1970). Primary 52-04, 52 A40.

Copyright $\odot 1977$, American Mathematical Society 
TABLE 1

\begin{tabular}{l|cccccc}
\hline Configuration & 5 & 6 & 7 & 8 & 9 & 10 \\
\hline regular $N$-gon & 15.3884 & 22.3923 & 30.6690 & 40.2187 & 51.0415 & 63.1375 \\
regular pyramid & 15.6748 & 22.8237 & 31.2447 & 40.9381 & 51.9041 & 64.1429 \\
regular bipyramid & 15.6814 & 22.9706 & 31.5306 & 41.3629 & 52.4680 & 64.8461 \\
prism & - & 22.9128 & - & 41.4523 & - & 65.2747 \\
skew prism & - & 22.9706 & - & 41.4731 & - & 65.2817 \\
computer search & 15.6814 & 22.9706 & 31.5309 & 41.4731 & 52.7436 & 65.3497 \\
$(2 / 3) N^{2}-1 / 2$ & 16.1667 & 23.5 & 32.1667 & 42.1667 & 53.5 & 66.1667 \\
\hline
\end{tabular}

In the first row are the numerical values of the formula of Fejes Tóth mentioned above. The regular $N$-pyramid is a right pyramid with a regular $(N-1)$-gon base and a height chosen to optimize $S(N)$. The regular $N$-bipyramid has a point at the North and South poles and a regular $(N-2)$-gon in the equatorial plane. The prism listed in the fourth row has $N$ vertices, with regular polygons for its bases, and optimal height. The skew prism is a prism with one of its bases rotated through $2 \pi / N$ radians, and height optimal. The final row represents the upper bound for $S(N)$ given in [1]

For $N=5,6$ our computer search gave the regular bipyramid as the optimal configuration. For $N=8$ a skew square-based prism was obtained (i.e. an inscribed square-based prism with the top square twisted through $45^{\circ}$ ). The bases have edge length 1.1633 , and the planes of the bases are at a distance of 1.1373 . With $N=9$ the configuration is an equilateral triangle in the equatorial plane and equilateral triangles above and below this plane at a height of .7031. These smaller triangles are skewed $60^{\circ}$ with respect to the equatorial triangle. For $N=10$ the algorithm produced a point at each pole and a skew square-based prism in between.

TABLE 2

\begin{tabular}{l|ccccccc}
\hline & 1 & 2 & 3 & 4 & 5 & 6 & 7 \\
\cline { 2 - 7 } 1 & 0.00000 & 1.56030 & 1.31347 & 1.43770 & 1.42931 & 1.31681 & 1.99914 \\
2 & 1.56030 & 0.00000 & 1.18070 & 1.88124 & 1.88478 & 1.18164 & 1.29635 \\
3 & 1.31347 & 1.18070 & 0.00000 & 1.19471 & 1.91488 & 1.86137 & 1.52339 \\
4 & 1.43770 & 1.88124 & 1.19471 & 0.00000 & 1.20434 & 1.91690 & 1.35798 \\
5 & 1.42931 & 1.88478 & 1.91488 & 1.20434 & 0.00000 & 1.19618 & 1.36356 \\
6 & 1.31681 & 1.18164 & 1.86137 & 1.91690 & 1.19618 & 0.00000 & 1.51618 \\
7 & 1.99914 & 1.29635 & 1.52339 & 1.35798 & 1.36356 & 1.51618 & 0.00000 \\
\hline
\end{tabular}


In the case $N=7$ the computer search produced a configuration close to the regular 7-bipyramid. It consists of two points almost antipodal and the remaining five points sprinkled around an equatorial band. In the table of mutual distances above, points 1 and 7 are the almost antipodal points. The sum of the mutual distances of the points of this figure improve by $.001 \%$ that of the regular 7-bipyramid. Moreover, the vector sum of the 7 points is not at the origin.

We note that with the exception of $N=7$, the configurations which arose in our computer search closely agree with the known best solutions for minimizing the total potential of unit charges on the sphere, i.e. minimizing $\Sigma 1 /\left|p_{i}-p_{j}\right|$. Consult [5] or [7] for details. Also, [10] contains a detailed discussion of these and related problems.

Department of Mathematics

University of Illinois at Chicago Circle

Chicago, Illinois 60680

Department of Mathematics

Eastern Washington State College

Cheney, Washington 99004

1. J. R. ALEXANDER, "On the sum of distances between $N$ points on a sphere," Acta Math. Acad. Sci. Hungar., v. 23, 1972, pp. 443-448.

2. J. R. ALEXANDER \& K. B. STOLARSKY, "Extremal problems of distance geometry related to energy integrals," Trans. Amer. Math. Soc., v. 193, 1974, pp. 1-31. MR 50 \# 3121.

3. J. D. BERMAN \& KIT HANES, "Volumes of polyhedra inscribed in the unit sphere in $E^{3}$," Math. Ann., v. 188, 1970, pp. 78-84. MR $42 \# 961$.

4. G. D. CHAKERIAN \& M. S. KLAMKIN, "Inequalities for sums of distances," Amer.

Math. Monthly, v. 80, 1973, pp. 1009-1017. MR 48 \#958.

5. H. COHN, "Stability configurations of electrons on a sphere," MTAC, v. 10, 1956, pp. 117-120. MR 18, 356.

6. L. FEJES-TÓTH, “On the sum of distances determined by a pointset," Acta Math. Acad. Sci. Hungar., v. 7, 1956, pp. 397-401. MR 21 \# 5937.

7. M. GOLDBERG, "Stability configurations of electrons on a sphere," Math. Comp., v. 23, 1969, pp. $785-786$.

8. D. W. GRACE, “Search for largest polyhedra," Math. Comp., v. 17, 1963, pp. 197-199.

9. E. HILLE, "Some geometric extremal problems," J. Austral. Math. Soc., v. 6, 1966, pp. 122-128. MR 33 \# 6507.

10. T. W. MELNYK, O. KNOP \& W. R. SMITH, "Extremal arrangements of points and unit charges on a sphere: equilibrium configurations revisited," Canad. J. Chem., v. 55, 1977, pp. 17451761.

11. K. B. STOLARSKY, "The sum of the distances to $N$ points on a sphere," Pacific J. Math., v. 57, 1975, pp. 563-573. MR 53 \# 284.

12. K. B. STOLARSKY, "Sums of distances between points on a sphere. II," Proc. Amer. Math. Soc., v. 41, 1973, pp. 575-582. MR 48 \# 12314. 